International Journal of Pure and Applied Mathematics

Volume 103 No. 4 2015, 667-673

ISSN: 1311-8080 (printed version); ISSN: 1314-3395 (on-line version)

url: http://www.ijpam.eu

doi: http://dx.doi.org/10.12732/ijpam.v103i4.6

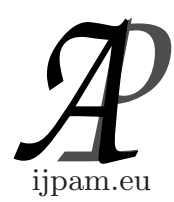

\title{
SOLUTION OF SOME CONFORMABLE FRACTIONAL DIFFERENTIAL EQUATIONS
}

Roshdi Khalil ${ }^{1} \S$, Heba Abu-Shaab ${ }^{2}$

${ }^{1,2}$ The University of Jordan

Al Jamia Al Urduniyya, Amman, JORDAN

\begin{abstract}
In this paper, we solve some conformable fractional partial differential equations including the wave equation. While the solution of such equations is often either numerical or too long, in our case we use fractional Fourier series and separation of variables, where solutions turned out to be easy and interesting.
\end{abstract}

AMS Subject Classification: 26A33

Key Words: conformable fractional derivative

\section{Introduction}

In [4], a new definition called conformable fractional derivative was introduced. For $0<\alpha \leq 1$, we set

$$
T_{\alpha}(f) x=f^{(\alpha)}(x)=\lim _{\epsilon \rightarrow 0} \frac{f\left(x+\epsilon x^{1-\alpha}\right)-f(x)}{\epsilon} .
$$

It turned out that this definition is very easy to handle and helped to solve easily many fractional differential equations.

We list here the conformable fractional derivatives of certain functions:

$$
\begin{gathered}
T_{\alpha}\left(t^{p}\right)=p t^{p-\alpha}, \quad T_{\alpha}\left(\sin \frac{1}{\alpha} t^{\alpha}\right)=\cos \frac{1}{\alpha} t^{\alpha} \\
T_{\alpha}\left(\cos \frac{1}{\alpha} t^{\alpha}\right)=-\sin \frac{1}{\alpha} t^{\alpha}, \quad \operatorname{and} T_{\alpha}\left(e^{\frac{1}{\alpha} t^{\alpha}}\right)=e^{\frac{1}{\alpha} t^{\alpha}} .
\end{gathered}
$$

Letting $\alpha=1$ in these derivatives, we get the corresponding ordinary derivatives.

Received: April 23, 2015

(c) 2015 Academic Publications, Ltd.

$\S$ Correspondence author url: www.acadpubl.eu 
One should notice that a function could be $\alpha$-differentiable at a point but not differentiable, for example, take $f(t)=2 \sqrt{t}$. Then $T_{\frac{1}{2}}(f)(t)=1$. Hence $T_{\frac{1}{2}}(f)(0)=1$. But $T_{1}(f)(0)$ does not exist. This is not the case for the known classical fractional derivatives. In this paper we solve two well known partial fractional differential equations using separation of variables and Fractional Fourier series introduced in [7]. We refer to [1], [2], [3], [5], and [6] for more on different types of fractional derivatives.

\section{Fractional Heat Partial Differential Equation}

In this section we solve the Conformable Fractional heat partial differential equation.

Let us discuss the solution of the following fractional heat partial fractional differential equation:

$$
\begin{gathered}
\frac{\partial^{\alpha}}{\partial t^{\alpha}}\left(\frac{\partial^{\alpha} u(x, t)}{\partial t^{\alpha}}\right)=c^{2} \frac{\partial^{2} u(x, t)}{\partial x^{2}}, \quad t>0,0<x<L, \\
u(0, t)=0, \\
u(L, t)=0, \\
\frac{\partial u}{\partial x}(0, t)=t^{2 \alpha}
\end{gathered}
$$

and

$$
u(x, 0)=0 .
$$

Let $u(x, t)=P(x) Q(t)$.Substituting in the differential equation to get

$$
\frac{d^{\alpha}}{d t^{\alpha}} \frac{d^{\alpha} Q(t)}{d t^{\alpha}} P(x)=c^{2} Q(t) \frac{d^{2} P(x)}{d x^{2}} .
$$

Hence, for some constant $\lambda$ :

$$
\frac{d^{\alpha}}{d t^{\alpha}} \frac{d^{\alpha} Q(t)}{d t^{\alpha}} / Q(t)=c^{2} \frac{d^{2} P(x)}{d x^{2}} / P(x)=\lambda .
$$

Consequently

$$
\begin{aligned}
& \frac{d^{\alpha}}{d t^{\alpha}} \frac{d^{\alpha} Q(t)}{d t^{\alpha}}-\lambda Q(t)=0, \\
& c^{2} \frac{d^{2} P(x)}{d x^{2}}-\lambda P(x)=0 .
\end{aligned}
$$


We start with equation $(7), \frac{d^{2} P(x)}{d x^{2}}-\frac{\lambda}{c^{2}} P(x)=0$. Then the auxiliary equation of equation (7) is $r^{2}-\frac{\lambda}{c^{2}}=0$. Now:

1. $\lambda=0$, so $r=0$, then the solution is $P(x)=c_{1}+c_{2} x$.Using condition (2), we get $c_{1}=0$. Again using condition (3), we get $c_{2}=0$, so $P(x)=0$.Hence there is no non trivial solution when $\lambda=0$.

2. $\lambda>0$, say $\lambda=\mu^{2}$, so $r=+\frac{\mu}{c}, r=-\frac{\mu}{c}$, then the solution is $P(x)=$ $c_{1} \sinh \frac{\mu}{c} x+c_{2} \cosh \frac{\mu}{c} x$. Using condition (2) and (3), we get $c_{1}=0$ and $c_{2}=0$,so $P(x)=0$. Hence there is no non trivial solution when $\lambda>0$.

3. $\lambda<0$, say $\lambda=-\mu^{2}$,so $r= \pm i \frac{\mu}{c}$ then the solution is $P(x)=c_{1} \sin \frac{\mu}{c} x+$ $c_{2} \cos \frac{\mu}{c} x$. Using condition (2), we get $c_{2}=0$. Using condition (3), we get $P(L)=$ $c_{1} \sin \frac{\mu}{c} L=0$, so $\sin \frac{\mu}{c} L=0$.

Hence, $\mu=\frac{c}{L} n \pi, n=1,2,3, \ldots$. which gives that $\lambda=-\frac{c^{2}}{L^{2}} n^{2} \pi^{2}, n=$ $1,2,3, \ldots$ Thus

$$
P_{n}(x)=c_{n} \sin \left(\frac{n \pi}{L} x\right) .
$$

Now, we return back to equation (6) $\frac{d^{\alpha}}{d t^{\alpha}} \frac{d^{\alpha} Q(t)}{d t^{\alpha}}-\lambda Q(t)=0$. which is equivalent to $\frac{d^{\alpha}}{d t^{\alpha}} \frac{d^{\alpha} Q(t)}{d t^{\alpha}}=\lambda Q(t)$.

Using formulas $T_{\alpha}\left(\cos \frac{t^{\alpha}}{\alpha}\right)=-\sin \frac{t^{\alpha}}{\alpha}$, and $T_{\alpha}\left(\sin \frac{t^{\alpha}}{\alpha}\right)=\cos \frac{t^{\alpha}}{\alpha}$. We get $Q(t)=b_{1} \cos \left(\frac{c}{L} n \pi \frac{t^{\alpha}}{\alpha}\right)+b_{2} \sin \left(\frac{c}{L} n \pi \frac{t^{\alpha}}{\alpha}\right)$. Condition (5) implies that $b_{1}=0$. So $Q(t)=b_{2} \sin \mu \frac{t^{\alpha}}{\alpha}$. Hence

$$
Q_{n}(t)=b_{n} \sin \left(n \pi \frac{c}{L} \frac{t^{\alpha}}{\alpha}\right)
$$

Combining (8) and (9) to get

$$
u(x, t)=\sum_{n=1}^{\infty} a_{n} \sin \left(\frac{n \pi}{L} x\right) \sin \left(n \pi \frac{c}{L} \frac{t^{\alpha}}{\alpha}\right) .
$$

Now, using condition (4), $\frac{\partial u}{\partial x}(0, t)=t^{2 \alpha}$, to get

$$
t^{2 \alpha}=\sum_{n=1}^{\infty} \frac{n \pi}{L} a_{n} \sin \left(n \pi \frac{c}{L} \frac{t^{\alpha}}{\alpha}\right) .
$$

Using the $\alpha$-Fractional Fourier series of $t^{2 \alpha}$, we find that

$$
a_{n}=\frac{2 \alpha}{p^{\alpha}} \int_{0}^{p} t^{2 \alpha} \sin \left(n \frac{t^{\alpha}}{\alpha}\right) \frac{d t}{t^{1-\alpha}}=\frac{2 \alpha}{p^{\alpha}} \int_{0}^{p} t^{3 \alpha-1} \sin \left(n \frac{t^{\alpha}}{\alpha}\right) d t .
$$


By using the substitution $u=t^{\alpha}$, and $d u=\alpha t^{\alpha-1} d t$, then

$$
\begin{aligned}
a_{n}=\frac{2 \alpha}{p^{\alpha}} \int_{0}^{p^{\alpha}} t^{3 \alpha-1} \sin \left(n \frac{u}{\alpha}\right) \frac{t^{1-\alpha}}{\alpha} d u=\frac{2}{p^{\alpha}} \int_{0}^{p^{\alpha}} t^{2 \alpha} \sin \left(n \frac{u}{\alpha}\right) d u, & \\
a_{n} & =\frac{2}{p^{\alpha}} \int_{0}^{p^{\alpha}} u^{2} \sin \left(n \frac{u}{\alpha}\right) d u .
\end{aligned}
$$

Consequently

$$
\begin{gathered}
a_{n}=\frac{2}{p^{\alpha}}\left[-u^{2} \cos \left(\frac{n}{\alpha} u\right) \cdot \frac{\alpha}{n}+2 u \sin \left(\frac{n}{\alpha} u\right) \cdot \frac{\alpha^{2}}{n^{2}}+2 \cos \left(\frac{n}{\alpha} u\right) \frac{\alpha^{3}}{n^{3}}\right]_{0}^{p^{\alpha}}, \\
a_{n}=\frac{2}{p^{\alpha}}\left[-p^{2 \alpha} \cos \left(\frac{n}{\alpha} p^{\alpha}\right) \cdot \frac{\alpha}{n}+2 p^{\alpha} \sin \left(\frac{n}{\alpha} p^{\alpha}\right) \cdot \frac{\alpha^{2}}{n^{2}}+2 \cos \left(\frac{n}{\alpha} p^{\alpha}\right) \cdot \frac{\alpha^{3}}{n^{3}}\right]-2 \frac{\alpha^{3}}{n^{3}}, \\
a_{n}=-2 p^{\alpha} \cos \left(\frac{n}{\alpha} p^{\alpha}\right) \cdot \frac{\alpha}{n}+4 \sin \left(\frac{n}{\alpha} p^{\alpha}\right) \cdot \frac{\alpha^{2}}{n^{2}}+\frac{4}{p^{\alpha}} \cos \left(\frac{n}{\alpha} p^{\alpha}\right) \cdot \frac{\alpha^{3}}{n^{3}}-2 \frac{\alpha^{3}}{n^{3}} .
\end{gathered}
$$

Therefore

$$
u(x, t)=\sum_{n=1}^{\infty} a_{n} \sin \left(\frac{n \pi}{L} x\right) \sin \left(n \pi \frac{c}{L} \frac{t^{\alpha}}{\alpha}\right)
$$

Here

$$
a_{n}=-2 p^{\alpha} \cos \left(\frac{n}{\alpha} p^{\alpha}\right) \cdot \frac{\alpha}{n}+4 \sin \left(\frac{n}{\alpha} p^{\alpha}\right) \cdot \frac{\alpha^{2}}{n^{2}}+\frac{4}{p^{\alpha}} \cos \left(\frac{n}{\alpha} p^{\alpha}\right) \cdot \frac{\alpha^{3}}{n^{3}}-2 \frac{\alpha^{3}}{n^{3}} .
$$

\section{Fractional Diffusion Wave Partial Differential Equation}

Let us discuss the solution of the following conformable fractional diffusion wave partial differential equation

$$
\frac{\partial^{2 \alpha} u(x, t)}{\partial x^{2 \alpha}}=-k \frac{\partial^{2} u(x, t)}{\partial t^{2}}, \quad 0<x<L, t \geq 0, \text { and } 0<\alpha \leq 1,
$$

subjected to the conditions

$$
\begin{aligned}
& u(0, t)=0, \\
& u(L, t)=0,
\end{aligned}
$$




$$
\begin{aligned}
u(x, 0) & =0, \\
\frac{\partial}{\partial t} u(x, 0) & =f(x) .
\end{aligned}
$$

Let $u(x, t)=P(x) Q(t)$. Substituting in the differential equation to get

$$
\frac{d^{2 \alpha} P(x)}{d x^{2 \alpha}} Q(t)=k P(x) \frac{d^{2} Q(t)}{d t^{2}} .
$$

Hence, for some constant $\lambda$ we have $\frac{d^{2 \alpha} P(x)}{d x^{2 \alpha}} / P(x)=-k \frac{d^{2} Q(t)}{d t^{2}} / Q(t)=\lambda$.

Consequently

$$
\begin{aligned}
& \frac{d^{2 \alpha} P(x)}{d x^{2 \alpha}}-\lambda P(x)=0, \\
& \frac{d^{2} Q(t)}{d t^{2}}+\frac{\lambda}{k} Q(t)=0 .
\end{aligned}
$$

Conditions (2) and (3) suggest that we work with equation (6) first. There are three possibilities for $\lambda$ :

1. $\lambda=0$. Then equation (6) becomes $\frac{d^{2 \alpha} P(x)}{d x^{2 \alpha}}=0$. And from the property $T_{\alpha}(c)=0$ of the conformable fractional derivative, we get $P(x)=c$. Conditions (2) and (3) show that $c=0$.So no non-trivial solution exists when $\lambda=0$.

2. $\lambda>0$. Then equation (6) becomes $\frac{d^{2 \alpha} P(x)}{d x^{2 \alpha}}=\lambda P(x)$. And from the property $T_{\alpha}\left(e^{\frac{t^{\alpha}}{\alpha}}\right)=e^{\frac{t^{\alpha}}{\alpha}}$ of the conformable fractional derivative, we get $P(x)=$ $c e^{\sqrt{\lambda} \frac{x^{\alpha}}{\alpha}}$. But again condition (2) and (3) show that $c=0$,so no non-trivial solution when $\lambda>0$.

3. $\lambda<0$. Then equation (6) becomes $\frac{d^{2 \alpha} P(x)}{d x^{2 \alpha}}+\mu^{2} P(x)=0$. Using formulas $T_{\alpha}\left(\cos \frac{t^{\alpha}}{\alpha}\right)=-\sin \frac{t^{\alpha}}{\alpha}$ and $T_{\alpha}\left(\sin \frac{t^{\alpha}}{\alpha}\right)=\cos \frac{t^{\alpha}}{\alpha}$ of the conformable fractional derivative. We get

$$
P(x)=c_{1} \cos \mu \frac{x^{\alpha}}{\alpha}+c_{2} \sin \mu \frac{x^{\alpha}}{\alpha} .
$$

Condition (2) implies that $c_{1}=0$. So $P(x)=c_{2} \sin \mu \frac{x^{\alpha}}{\alpha}$. Condition (3) implies that $\sin \mu \frac{L^{\alpha}}{\alpha}=0$. Hence $\mu=n \pi \frac{\alpha}{L^{\alpha}}$, with $n=1,2, \ldots$.

So

$$
P_{n}(x)=c_{n} \sin n \pi \frac{x^{\alpha}}{L^{\alpha}} .
$$
Hence

Now, we return back to equation (7) which becomes $\frac{d^{2} Q(t)}{d t^{2}}-\frac{\mu^{2}}{k} Q(t)=0$.

$$
Q(t)=a_{1} e^{\frac{n \pi \alpha}{L^{\alpha} \sqrt{k}} t}+a_{2} e^{-\frac{n \pi \alpha}{L^{\alpha} \sqrt{k}} t} .
$$


Condition (4) implies that $a_{2}=-a_{1}$. Hence

$$
Q(t)=2 a_{1} \sinh \frac{n \pi \alpha}{L^{\alpha} \sqrt{k}} t
$$

Combining (9) and (11) to get

$$
u(x, t)=\sum_{n=1}^{\infty} A_{n} \sin n \pi \frac{x^{\alpha}}{L^{\alpha}} \sinh \frac{n \pi \alpha}{L^{\alpha} \sqrt{k}} t .
$$

Now, using condition (5), we get $A_{n} \frac{n \pi \alpha}{L^{\alpha} \sqrt{k}}$ to be then fractional Fourier coefficients of the function $f$ we get $f(x)=\sum_{n=1}^{\infty} A_{n} \frac{n \pi \alpha}{L^{\alpha} \sqrt{k}} \sin n \pi \frac{x^{\alpha}}{L^{\alpha}}$.

Using the $\alpha 0$-Fourier sine series (see M. Abu Hammad, R. Khalil, 2014)

$$
A_{n} \frac{n \pi \alpha}{L^{\alpha} \sqrt{k}}=\frac{2 \alpha}{p^{\alpha}} \int_{0}^{p} f(x) \sin \left(n \frac{x^{\alpha}}{\alpha}\right) \frac{d x}{x^{1-\alpha}} .
$$

So

$$
A_{n}=\frac{1}{\frac{n \pi \alpha}{L^{\alpha} \sqrt{k}}} \frac{2 \alpha}{p^{\alpha}} \int_{0}^{p} f(x) \sin \left(n \frac{x^{\alpha}}{\alpha}\right) \frac{d x}{x^{1-\alpha}}
$$

Therefore

$$
u(x, t)=\sum_{n=1}^{\infty} A_{n} \sin n \pi \frac{x^{\alpha}}{L^{\alpha}} \sinh \frac{n \pi \alpha}{L^{\alpha} \sqrt{k}} t
$$

Here

$$
A_{n}=\frac{1}{\frac{n \pi \alpha}{L^{\alpha} \sqrt{k}}} \frac{2 \alpha}{p^{\alpha}} \int_{0}^{p} f(x) \sin \left(n \frac{x^{\alpha}}{\alpha}\right) \frac{d x}{x^{1-\alpha}}
$$

\section{References}

[1] K.S. Miller, An Introduction to Fractional Calculus and Fractional Differential Equations, J. Wiley and Sons, New York, 1993.

[2] K. Oldham, J. Spanier, The Fractional Calculus, Theory and Applications of Differentiation and Integration of Arbitrary Order, Academic Press, 1974. 
[3] A. Kilbas, H. Srivastava, J. Trujillo, Theory and Applications of Fractional Equations, Math. Studies. Northholland, New York, 2006.

[4] R. Khalil, M. Al Horani, A. Yousef, M. Sababheh, A new definition of fractional derivative, J. Comput. Appl. Math., 264 (2014), 6570.

[5] T. Abdeljawad, On conformable fractional calculus, J. Comput. Appl. Math., 279 (2015), 57-66.

[6] M. Abu Hammad, R. Khalil, Conformable fractional heat differential equation, International Journal of Pure and Applied Mathematics, 94 (2014), 215-2217.

[7] M. Abu Hammad, R. Khalil, Fractional Fourier series with applications, American Journal of Computational and Applied Mathematics, 4, No. 6 (2014), 187-191. 
